\title{
Differential Rotation and Magnetism in Simulations of Fully Convective Stars
}

\author{
Matthew K. Browning ${ }^{1}$ \\ ${ }^{1}$ Canadian Institute for Theoretical Astrophysics, \\ University of Toronto, 60 St. George St, Toronto, Canada \\ email: browning@cita.utoronto.ca
}

\begin{abstract}
Stars of sufficiently low mass are convective throughout their interiors, and so do not possess an internal boundary layer akin to the solar tachocline. Because that interface figures so prominently in many theories of the solar magnetic dynamo, a widespread expectation had been that fully convective stars would exhibit surface magnetic behavior very different from that realized in more massive stars. Here I describe how recent observations and theoretical models of dynamo action in low-mass stars are partly confirming, and partly confounding, this basic expectation. In particular, I present the results of 3-D MHD simulations of dynamo action by convection in rotating spherical shells that approximate the interiors of 0.3 solar-mass stars at a range of rotation rates. The simulated stars can establish latitudinal differential rotation at their surfaces which is solar-like at "rapid" rotation rates (defined within) and anti-solar at slower rotation rates; the differential rotation is greatly reduced by feedback from strong dynamo-generated magnetic fields in some parameter regimes. I argue that this "flip" in the sense of differential rotation may be observable in the near future. I also briefly describe how the strength and morphology of the magnetic fields varies with the rotation rate of the simulated star, and show that the maximum magnetic energies attained are compatible with simple scaling arguments.
\end{abstract}

Keywords. convection, MHD, stars: low-mass, stars: magnetic fields, stars: rotation, turbulence

\section{Introduction: Puzzles of Low-Mass Stellar Magnetism}

Magnetic fields are ubiquitous in low-mass stars, and in at least some cases those magnetic fields exhibit a remarkable amount of spatial and temporal organization. The most famous example is the Sun's cyclical magnetism: sunspots appear on the solar disk first at mid-latitudes, then progressively nearer the equator over the course of a roughly 11-year cycle; the number and polarity of the spots varies in the same way (see., e.g., Ossendrijver 2003). These organized magnetic fields are widely (though not universally) thought to be generated partly in the tachocline of shear at the base of the solar convective envelope - in part because it is a site of strong differential rotation, but also because the stable stratification below the convection zone might allow magnetic fields to be amplified enormously before becoming susceptible to magnetic buoyancy instabilities (see, e.g., Parker 1993; see Spruit 2010 for a different view).

But not all stars have a tachocline. Moving down the main sequence to lower masses and cooler temperatures, the convective envelope deepens and the radiative core shrinks. Stars of less than about a third a solar mass (corresponding to spectral types of roughly M3.5 or later) are thought to be convective throughout their interiors, and so do not possess an internal boundary layer akin to the solar tachocline. Although these lowmass stars might in principle still possess differential rotation - like the latitudinal shear present throughout the solar convection zone - a widespread theoretical expectation had 
been that they would exhibit magnetic fields that differed appreciably from those realized in more massive solar-like stars (e.g., Durney et al. 1993).

Observations of stellar magnetism paint a somewhat murkier picture. On the one hand, there have been recent suggestions (particularly from spectropolarimetric observations) that the surface magnetic topologies of stars with a small radiative core do differ appreciably from those of stars that are fully convective (e.g., Morin et al. 2010; Reiners \& Basri 2009). Fully convective stars (with late-M spectral types) also appear to spin down (through angular momentum loss via a magnetized stellar wind) much more slowly than early-M dwarfs (e.g., Browning et al. 2010; Reiners et al. 2009), though it is unclear whether this is due to changes in the strength or morphology of surface magnetic fields or to changes in the mass loss rate. On the other hand, it also seems clear that some fully convective stars can build magnetic fields with remarkably strong large-scale components (Donati et al. 2006), with the strength of those fields sensitive to rotation at some level (e.g., Mohanty \& Basri 2003). Indeed, some recent comparisons between line-of-sight and unsigned field measurements (extracted from Stokes V and Stokes I observations) suggest that some fully convective stars actually harbor more organized fields than slightly more massive stars with a small radiative core (Reiners \& Basri 2009). Puzzlingly, some of these stars appear to show large-scale magnetic field organization but no evident surface differential rotation (e.g., Donati et al. 2006).

The most central question raised by these observations is, simply, how are spatially organized fields realized in fully convective stars? But this basic issue is tightly linked to a whole set of other questions: what is the nature of the differential rotation in these stars, and what role does it play in the dynamo process? Can these stars ever drive solar-like differential rotation at observable levels? How do the strength and morphology of the dynamo-generated fields vary with rotation rate? How does the magnetism modify the transport of energy and angular momentum throught the stellar interior? These questions motivate the work described in this paper.

Here, I describe the results of a series of 3-D simulations of convection and magnetism in rotating spherical domains that are intended to represent fully convective stars of 0.3 solar masses at various rotation rates. In $\S 2$ I describe the basic setup of these simulations and the numerical methods employed. The convective flow patterns and some aspects of energy transport are detailed in $\S 3$. I describe the differential rotation that arises in these models in $\S 4$. In $\S 5$ I describe the strength and morphology of the magnetic fields realized at various rotation rates.

\section{Model Formulation and Numerical Methods}

The simulations described here are highly idealized representations of 0.3 solar-mass stars rotating between one-tenth and ten times as rapidly as the Sun $\left(0.1-10 \times \Omega_{0}=\right.$ $\left.2.6 \times 10^{-6} \mathrm{~s}^{-1}\right)$. They were all carried out using the Anelastic Spherical Harmonic (ASH) code, which solves the $3-\mathrm{D}$ Navier-Stokes equations with magnetism in the anelastic approximation (Clune et al. 1999; Miesch et al. 2000; Brun, Miesch \& Toomre 2004). The setup at each rotation rate is essentially identical to that described in Browning (2008); here I summarize only the most important details. Before delving into them, note that the ASH code is well-suited to this problem because it correctly captures the global spherical geometry of the star, thereby allowing the study of intrinsically largescale processes like differential rotation, meridional circulation, and global-scale dynamo action. The tradeoff, of course, is that because we can only resolve a finite range of spatial and temporal scales in the simulation, including the largest possible length scales (the radius of the star) in our modeling implies that the smallest lengths resolved are still quite 
large - typically about $1 \mathrm{Mm}$. Thus I focus here on the dynamics of the largest scales, while recognizing that at some level of detail these must be influenced by smaller-scale dynamics that these simulations cannot resolve.

The spherical computational domain typically extends from $0.06-0.96 \mathrm{R}$, where $R$ is the overall stellar radius of $2.07 \times 10^{10} \mathrm{~cm}$, thus excluding both the surface boundary layer and the innermost portions of the star. I exclude the inner few percent of the star from these calculations both because the coordinate systems employed in ASH are singular there, and because the small numerical mesh sizes at the center of the star would require impractically small timesteps. The initial stratifications of the mean density, energy generation rate, gravity, radiative diffusivity, and entropy gradient $d S / d r$ are adopted from a 1-D stellar model (I. Baraffe, private communication, after Baraffe \& Chabrier 2003). These thermodynamic quantities are updated throughout the course of the simulation as the evolving convection modifies the spherically symmetric mean state. Variables are expanded in terms of spherical harmonic basis functions $Y_{l}^{m}(\theta, \phi)$ in the horizontal directions and Chebyshev polynomials $T_{n}(r)$ in the radial. As with all numerical simulations, the eddy viscosities and diffusivities employed are vastly greater than their counterparts in actual stars; here I have taken these to be constant in radius, and adopted a Prandtl number $\nu / \kappa=0.25$ and a magnetic Prandtl number $P m=\nu / \eta$ that varies between 0.25 and 8 depending on the simulation. At a detailed level, the flows and magnetic fields attained in the simulations are sensitive to the values of these nondimensional numbers. Because of this, I focus here on the broad trends these simulations exhibit: the types of flows and magnetic fields they can achieve as the basic parameters of the problem are varied, rather than the precise values of magnetic energy, zonal wind velocity, etc, that are attained. I particularly concentrate here on the role that changes in the overall stellar rotation rate play, since this turns out to have an outsized influence on the flows and magnetic fields that are achieved.

\section{Convective Flows and Energy Transport}

The convective flows in these simulations possess structure on many scales. Although many small-scale features continually emerge, propagate, and survive only for a short while, there are also large-scale organized motions that can persist for extended intervals. A sampling of this behavior is provided by Figure 1, which shows volume renderings of the radial velocity in two simulations, one rotating at the solar angular velocity and the other ten times slower. (The opacity mapping used in that figure is such that only motions near
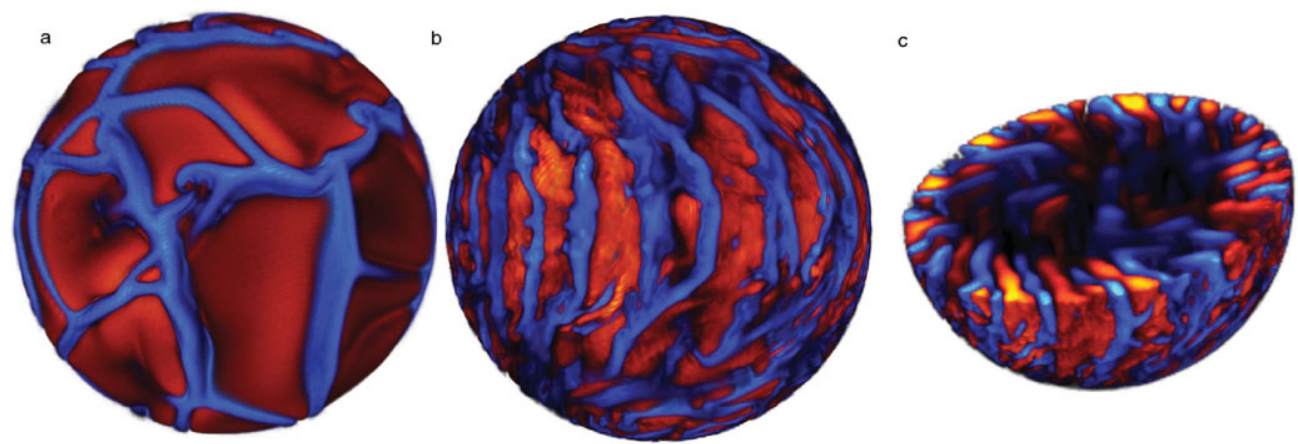

Figure 1. Radial velocity in simulations rotating at the solar angular velocity $(b, c)$ and tenfold slower $(a)$. Upflows are reddish (light), downflows are blueish (dark). Panel $c$ shows a cutaway of one hemisphere. 
the outer boundary of the simulation are visible in Fig. $1 a, 1 b$; Figure $1 c$ shows a cutaway of one hemisphere in the more rapidly rotating simulation, to highlight the radial extent of the motions.) Rotation has a strong effect on the convective patterns that are realized: when rotation is very slow, the convection is more or less isotropic on each spherical surface, with a network of upflows and downflows that meanders in orientation over the sphere. Increasing the rotation rate breaks this symmetry and imposes a preferred direction, leading (in some cases) to convective rolls like those shown in Figure $1 b, 1 c$. (If the eddy viscosity in the simulations is decreased, these rolls become less pronounced and can break up, but there is still a tendency towards alignment along the rotation axis.) These roll-like structures are a well-known feature of convection in rotating spherical shells (see, e.g., Busse 2002; Gilman 1977).

One other feature worth highlighting is the strong asymmetry between upflows and downflows realized near the top of the simulated stars: the downflows are stronger and narrower than the former, mainly because of the strong density stratification. (Downflows are cool and contract; upflows are hot and expand.) Deeper in the interior, the flows are weaker and of somewhat larger physical scale: motions can span large fractions of a hemisphere and extend radially for some distance. Flow amplitudes also vary appreciably with depth, with typical rms velocities declining by a factor of about ten in going from the surface to the center.

The variation in flow amplitude with radius is linked to both the density stratification and to radial variations in the amount of energy that must be transported by convection. Convection ultimately arises because of a need to transport heat outwards: if more energy has to be carried by convection, the convective velocity will generally be higher. Although the interior is unstably stratified everywhere, radiation still carries some of the energy at small radii. This is because the end state of efficient convection is an interior stratification that is approximately isentropic, not isothermal as in unstratified convection: thus there is still a non-zero radiative flux. Together with the overall increase of the total luminosity with radius (out to the point where nuclear energy generation stops), this implies that the total luminosity carried by convection peaks at large radii (around $r=0.80 R$ ). Thus the convective velocity is appreciably greater near the surface than at depth. Another important effect arises because of the asymmetry between upflows and downflows: this implies a negative (inward) kinetic energy flux, which in a steady state must be compensated for by an increased outward enthalpy (convective) flux. This effect, too, depends on depth (since the star is more strongly stratified near the surface), again leading to more vigorous convection near the top of the simulation domain.

\section{Differential Rotation: Solar or Anti-Solar}

In addition to transporting heat, the convective flows also transport angular momentum. One might naively expect that parcels of fluid would individually conserve angular momentum - so that (for instance) a fluid element moving outward would tend to slow down (move retrograde relative to the frame), and a fluid element moving latitudinally from equator to pole would tend to spin up. This would imply anti-solar differential rotation at the surface, with a slow equator and fast poles, and longitudinal velocities that decrease with distance from the rotation axis. The fact that the Sun in fact has a fast equator and slow pole is enough to suggest that there is more to the story than this: that convection, acting in concert or conflict with meridional circulations and magnetic fields, can redistribute angular momentum in surprising ways. Here I describe briefly the types of differential rotation that are achieved in these simulations at varying rotation rates, 
while deferring an in-depth analysis of the angular momentum transport to forthcoming work (Browning \& Miesch 2010).

All of the MHD calculations described here had hydrodynamical progenitor simulations. These all began in a state of uniform rotation, but convection quickly established interior rotation profiles that varied with radius and latitude. The resulting differential rotation, displayed in Figure 2 for two hydrodynamic cases, depends on the overall stellar rotation rate: in Figure $2 a$, from a simulation rotating at one-tenth the solar angular velocity, the rotation profile is "anti-solar" at the surface, with a slow equator and fast poles; in Figure $2 b$, from a simulation rotating at the solar angular velocity, the rotation profile is solar-like at the surface. Both cases also show angular velocity contrasts in radius, with angular velocity decreasing with depth in the more rapidly rotating case and decreasing with depth in the slower rotator. In the simulation rotating at the solar rate, the interior angular velocity profile is largely constant on cylinders, reflecting the strong Proudman-Taylor constraint; in the more slowly rotating case this cylindrical alignment is not evident.

Similar transitions in the nature of angular momentum transport and differential rotation have been noted by several previous authors in other contexts. In particular, Gilman (1977) noted that his simulations of a solar-like convection zone exhibited solar-like equatorial acceleration in some regimes and anti-solar rotation profiles in others; he identified the transition between these two regimes with a transition from a convective Rossby number (essentially the ratio of buoyancy driving to Coriolis forces) greater than unity to less than unity. When the rotational influence on the convection was strong relative to buoyancy driving, he attained equatorial acceleration; when it was weak, the equator rotated more slowly than the poles. Similar results were obtained in simulations of core convection in A-type stars (Browning et al. 2004). The simulations here exhibit qualitatively similar behavior. Convection influenced by rotation (that is, with a Rossby number roughly less than unity) tends to drive solar-like differential rotation; when rotation is slower, the differential rotation profile is anti-solar.

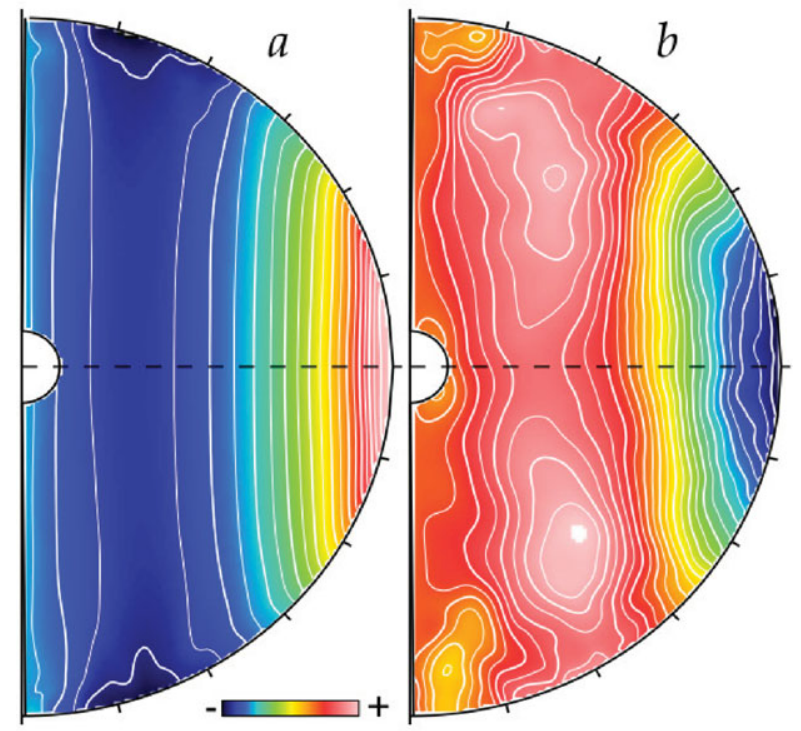

Figure 2. Differential rotation achieved in sample hydrodynamic simulations rotating at the solar angular velocity $(a)$ and ten times slower $(b)$. Light tones are prograde, dark ones retrograde. 
The interior rotation profiles can be quite different in the presence of strong dynamogenerated magnetic fields. In MHD simulations, the magnetic fields react back strongly upon the flows, acting to quench and in some instances essentially eliminate the differential rotation. An example of this for a simulation rotating at the solar rate was explored in Browning (2008). The extent to which the differential rotation is reduced depends on the overall magnetic field strength, which in turn (as discussed below) depends somewhat on the overall stellar rotation rate. Thus, there is a "sweet spot" for differential rotation: when the simulations rotate very slowly, they drive weak anti-solar differential rotation; when the rotation rate is somewhat larger they drive strong solar-like differential rotation; and when rotation is more rapid still they build magnetic fields so strong that the differential rotation is partly quenched. These transitions occur at fairly slow overall rotation rates: because the luminosities of M-dwarfs are so low, their convective velocities are also low, and so they are strongly influenced by rotation even at solar-like angular velocities. Only the very slowest rotators (those with rotation periods much longer than the solar value) drive anti-solar differential rotation.

It seems plausible that these basic predictions could be testable in the near future by photometric monitoring with, e.g., Kepler. The exact rotation periods at which stars might transition from anti-solar to solar-like differential rotation, and then again to no evident differential rotation (because it has been wiped out by the dynamo-generated Maxwell stresses), are probably not reliably predictable by these calculations. They depend on the values of viscosity, magnetic diffusivity, etc, adopted in the modeling. But the basic existence of these different regimes, and their rough dependence on rotation rate, might well be robust. The main challenge observationally will probably be to measure differential rotation reliably in the slowest rotators, which are presumably less active and so less spotted.

\section{Dynamo Action Achieved}

The flows in each simulation act as a magnetic dynamo, amplifying a tiny seed field by many orders of magnitude and sustaining it against Ohmic decay. The magnetic energy (ME) grows exponentially until it reaches a steady state. In the most rapid rotators (which, again, are not really rotating all that rapidly in absolute terms), the final magnetic energy density is approximately in equipartition with the flows; in slower rotators it tends to be smaller. The exact values of the magnetic energy are sensitive at some level to the values of the magnetic Reynolds number, magnetic Prandtl number, etc, that I have adopted in each calculation. To give a specific example: in an evolved calculation rotating at the solar angular velocity, ME was approximately $120 \%$ of the total kinetic energy KE (relative to the rotating frame) and about $140 \%$ of the convective (non-axisymmetric) kinetic energy (CKE).

As the fields grow, they react back on the flows through the Lorentz force. Thus KE begins to decline once ME reaches a threshold value of about $5 \%$ of KE; here this decline is associated mainly with a decrease in the energy of differential rotation DRKE, whereas CKE is less affected by the growing fields.

Like the flows that build them, the magnetic fields possess both intricate small-scale structure and substantial large-scale components. The typical length scale of the field increases with depth, partly tracking the radial variation in the size of typical convective flows. The smallest field structures are typically on finer scales than the smallest flow fields, partly because I have adopted a magnetic Prandtl number Pm greater than unity. By decomposing the magnetism into its azimuthal mean (TME), and fluctuations around that mean (FME), we can gain a coarse estimate of the typical size of field structures: if 
the field is predominantly on small scales, only a small signal will survive this azimuthal averaging. In these simulations, TME accounts for at most about $20 \%$ of the total magnetic energy in the bulk of the interior; it is smallest near the surface (where TME is typically less than $5 \% \mathrm{ME}$ ), and largest (as a fraction of ME) at depth.

The fraction of energy in the mean (axisymmetric) components increases with increasing rotation rate. The ratio of the toroidal mean energy to the poloidal mean also changes: in the very slowest rotators the two components are comparable, while in more rapidly rotating cases with strong differential rotation, TME exceeds the poloidal mean energy (PME) by a factor of a few.

The mean (axisymmetric) fields realized in some of the simulations are remarkably strong and long-lived. Mean toroidal field strengths can exceed $10 \mathrm{kG}$ in some locations; some prominent field structures persist for thousands of days. The overall field polarity is stable over long intervals (decades), in sharp contrast to some simulations of solar convection without a tachocline (Brun et al. 2004), in which the field polarity flipped at irregular intervals of less than 600 days.

\section{Closing Thoughts}

The overall picture that emerges from these simulations is that fully convective stars can act effectively as magnetic dynamos, building fields that have structure on both large and small spatial scales. The large-scale fields can be remarkably strong and longlived. The convection drives differential rotation, in a manner that depends on the overall rotation rate: the very slowest rotators would appear anti-solar at their surface, while more rapid rotators establish a fast equator and slow pole. That differential rotation is, however, largely quenched by Maxwell stresses in cases that build strong magnetic fields.

The maximum magnetic energy densities attained in these calculations are of order equipartition with the kinetic energy density relative to the rotating frame. Although stronger magnetic fields might be possible in some instances (see, e.g., Featherstone et al. 2009), it is worth noting that the assumption of equipartition yields magnetic field estimates broadly in line with those recently predicted on somewhat different grounds by Christensen and collaborators (see, e.g., Christensen et al. 2009; Reiners et al. 2009). Specifically, Reiners et al. (2009) argue, based on an energy flux scaling derived empirically from a series of planetary dynamo calculations, that the magnetic flux in stars should scale approximately as

$$
B f \propto M^{1 / 6} L^{1 / 3} R^{-7 / 6}
$$

with $M, L$, and $R$ the stellar mass, luminosity and radius, and where $B f$ is the surface magnetic flux. This turns out to be the same scaling one derives by assuming that 1 . magnetic fields are in approximate equipartition with the convective kinetic energy and 2 . that convective energy is given roughly by mixing-length arguments, so that it is proportional to the heat flux to the one-third power. To order of magnitude, the convective luminosity is given by

$$
L \sim \frac{\text { convective energy }}{\text { convective overturning time }} \sim \frac{\rho v^{2}\left(\frac{4}{3} \pi d^{3}\right)}{l_{c} / v}
$$

with $d$ a lengthscale characterizing the depth of the convection zone and $l_{c}$ the length characterizing convective eddies. This in turn implies that $v \sim\left(\frac{3 L}{4 \pi \rho} l_{c}\right)^{1 / 3} \frac{1}{d}$. Meanwhile, equipartition of kinetic and magnetic energy densities implies that $B \sim \rho^{1 / 2} v$. If we then take $\rho \sim M / R^{3}$, and assume that to order of magnitude all lengthscales are comparable 
$\left(l_{c} \sim d \sim R\right)$, we obtain that $v \sim L^{1 / 3} R^{1 / 3} M^{-1 / 3}$, so that finally $B \sim L^{1 / 3} M^{1 / 6} R^{-7 / 6}$, as in the Reiners et al. (2009) scaling. This is not to suggest that greater or lesser field energies are not possible, but does indicate that objects deviating greatly from the Reiners et al. (2009) scaling are breaking one of the assumptions I made above. Either equipartition does not hold, or the relevant flow velocity is not related to the background heat flux in the way assumed here.

It is a pleasure to acknowledge many helpful conversations about this and related puzzles in stellar rotation and magnetism with Juri and the other members of the "ASH mob."

\section{References}

Browning, M. K., 2008, ApJ, 676, 1262

Browning, M. K., Basri, G., Marcy, G. W., West, A. A., \& Zhang, J., 2010, AJ, 139, 504

Brun, A. S., Miesch, M. S., \& Toomre, J. 2004, ApJ, 614, 1073

Christensen, U. R., Holzwarth, V., \& Reiners, A., 2009, Nature, 457, 167

Donati, J. F., Forveille, T., Cameron, A. C., Barnes, J. R., Delfosse, X., Jardine, M. M., \& Valenti, J. A., 2006, Science, 311, 633

Durney, B. R., De Young, B. S., \& Roxburgh, I. W., 1993, Sol. Phys., 145, 207

Featherstone, N. A., Browning, M. K., Brun, A. S., \& Toomre, J., 2009, ApJ, 706, 1000

Gilman, P. A., 1977, GAPFD, 8, 93

Miesch, M. S., Elliott, J. R., Toomre, J., Clune, T. L., Gl atzmaier, G. A., \& Gilman, P. A., 2000, ApJ, 532, 593

Mohanty, S. \& Basri, G., 2003, ApJ, 583, 451

Morin, J., et al., 2010, MNRAS 1077

Ossendrijver, M. 2003, Astron. Astrophys. Rev., 11, 287

Parker, E. N. 1993, ApJ, 408, 707

Reiners, A. \& Basri, G., 2009, A\&A A, 496, 787

Reiners, A., Basri, G., \& Christensen, U. R., 2009, ApJ, 697, 373

Spruit, H. C. 2010, arXiv:1004.4545

\section{Discussion}

A. Brandenburg: Is the dependence of rotation rate on spectral type real and how does this depend on age?

M. Browning: The conclusion that very low-mass stars take longer to spin down than somewhat higher-mass ones appears to me to be quite robust. But there is certainly an age effect: if you look at young enough clusters, many stars of all spectral types are still rotating quite rapidly. In the field, though - i.e., looking at much older stars - you tend to find that very few early-M stars are detectably rotating (see, e.g., Browning et al. (2010), where we used Keck HIRES spectra to look for signs of rotation in dozens of field M-dwarfs). A significantly larger fraction of late-M stars (or L-dwarfs) are detectably rotating.

K. Moffat: Your conclusion that rotation plus convection are conducive to the growth of large-scale fields is hardly new! These ingredients imply large-scale helicity and so a corresponding $\alpha$-effect. Have you been able to interpret your simulations from this "mean-field" point of view?

M. Browning: The short answer is no, these simulations do not seem to be particularly well-described by mean-field theory. There is certainly large-scale helicity of the predicted sense, but it doesn't seem to be especially well-linked to the growth of the large-scale 
field. The role of rotation in these simulations seems to be partly about increasing the correlation length of the convection to something on the order of the domain size, rather than just imparting a preferred sign to the helicity in each hemisphere. That said, we certainly have more work to do in trying to make connections between these results and mean-field theory.

K. FERriere: Why in the Sun does the equator rotate faster than the poles?

M. Browning: I would say that, honestly, we still have a "description" of how this happens rather than a first-principles theory. (See Miesch et al., these proceedings, for some of that description.) Ultimately there are Reynolds stresses arising from correlations in the fluctuating velocity components, and the sense of those stresses is such that you break the tendency for individual parcels of fluid to just conserve angular momentum as they move outward or inward. But baroclinic effects, meridional circulations, etc, all appear to play roles as well. 